\title{
Fungus or Cancer: is it or is it not?
}

\author{
Sharon Murff* \\ Department of Nursing, Grambling State University, USA
}

*Corresponding author: Sharon Murff, Department of Nursing, Grambling State University School of Nursing, USA.

Received Date: March 07, 2019

Published Date: March 18, 2019

\begin{abstract}
Fungi are organisms that are ubiquitous. In fact, there are millions of different types of fungi that exist indoors and outdoors. "Some fungi do not cause serious problems and other species have the potential to be life-threatening and fungal infections can exist anywhere in the human body". Similarly, cancerous lesions can grow anywhere in the body and can increase an individual's mortality risk. There are radiographic character similarities between some fungal infections and cancerous lesions and there have been cases in which a misdiagnosis of cancer was given when in fact the problem was a fungal invasion. There is a vast difference in the treatment modalities for fungal infections and cancer and there is also a difference in the expense to manage patients. It is important to ensure an accurate diagnosis is provided in order to minimize any physiological, psychological, or financial challenges a patient might experience.
\end{abstract}

\section{Review}

The main focus of this review is to provide basic tenets associated with fungi, fungal infections, and cancer. Also, the chief aim of this review is to present information with regard to fungal infections that have been identified as malignancies from a radiologic perspective. Fungi exist in the environment and can cause health problems for anyone. Various fungal species can be found in the outdoor environment on plants, trees, in the soil, and in all buildings on various surfaces. Because fungi are ubiquitous it is difficult to escape contact with them [1].

Some fungal infections pose problems that are not serious while others might have devastating effects on the body. Common fungal infections often manifest on the skin, fingernails and toenails, and also might present as vaginal yeast infections. Alternately, some fungi pose life-threatening problems such as Aspergillosis, Blastomycosis, or Pneumocystis pneumonia [1].

Serious fungal infections have been pervasive among individuals who are immunosuppressed from medications or debilitating health conditions. Individuals with a compromised immune system include, but are not limited to, those with Acquired Immune Deficiency Syndrome (AIDS), malignancies, and various blood dyscrasias [2]. Other individuals who are vulnerable hosts for fungal infections include transplant recipients and those with cancer [2].
Sometimes fungi are called opportunistic in that they invade and infect the most vulnerable hosts and might also show up as a mass or tumor anywhere in the body [3]. Often fungal infections can be eradicated by non-drastic measures such as medication, or if the infection is in the lungs, an endobronchial procedure might be necessary [3]. On the other hand, a diagnosis of cancer has a morbid connotation and management carries a financial burden. The cost to care for someone with cancer might include surgery, radiotherapy, chemotherapy, palliative care, or more than one treatment modality [4].

Cancer is a name given to a group of diseases and it exists when cells in the body divide and multiply in an abnormal fashion. In 2018 the world-wide mortality related to cancer was over nine million and was ranked as the second leading cause of death worldwide [4]. The pathogenesis of cancer is ominous in that it can begin anywhere in the body and can develop into a solid form, as a tumor or mass, or non-solid form within the blood. In addition, cancer cells can be proliferative and can then be transported to invade any part of the body [5].

Tumors that stem from cells in the body have been described as malignant or benign. A malignant tumor is one that can spread and intrude into other tissue of the body in nearby spaces. In other words, malignant cells create a type of microenvironment in which 
normal cells can be channeled or recruited to support the growth of cancerous tumors (e.g. supportive blood supply) [5]. On the other hand, a benign tumor is one that does not usually have the potential to spread and cause harmful effects. Also, benign tumors, which are solid masses, do not usually re-emerge once they have been removed from the body through surgery or other technological procedures [4].

Fungi can produce masses that resemble malignancies on radiographs. "Researchers have reported radiologic findings in the pulmonary system that indicated a mass or lesion that appeared to be cancer [6]". Some fungi have been known to resemble primary lung cancer, based on radiologic findings, but after histological studies the final diagnosis was that of Aspergillosis, Mycormycosis, or Blastomycosis [6]. In the case of a 56 year old female who presented with a history of exposure to two brothers with Coccidicidomycosis, the radiograph indicated a mass in the right upper lobe of the lungs. Because the radiologic findings (computed tomography [CT]) of the female showed characteristics of a pulmonary malignancy, there was an initial misdiagnosis of a malignancy. The researchers, Guimaraes, Marchiori, and Godoy, stressed the importance of early detection of fungal disease of the lung due to the vast difference in management of fungal infections and malignancies [7].

In 2013, researchers reported that some fungal infections closely resembled malignant masses in several areas of the body. Results of a retrospective study in which the CT scans of 27 patients were examined, and the indications were that of a solid nodule or mass, and in some cases, patients were thought to have both a nodule and a mass. The chief, non-specific complaints of the patients included cough, chest pain, weakness, and some patients had hemoptysis, dyspnea, and fever. The initial diagnosis given to the patients in the study was that of cancer. The initial diagnosis, for some patients, included that of malignancies of the breast, liver, lung, esophagus, and thyroid gland. Also, lymphoma, leukemia, melanoma, and colorectal carcinoma diagnoses were given based on initial CT findings [8].

Histopathological analysis was performed on the patients in the study and the results were indicative of fungal infections. Approximately $50 \%$ of the patients in the study who were initially diagnosed with a malignancy, based on CT scan examination, had a final diagnosis of a fungal infection. Consequently, all patients with fungal infections were treated with antifungal medication and the follow-up CT scans indicated improvement or resolution of the infection [8].

\section{Conclusion}

In conclusion, there is a drastic difference between the treatment options for fungal infections and malignancies. Medical institutions and healthcare providers must address the rising expenses for health care along with an overall reduction in sufficient resources to provide adequate care. Therefore, patients must be provided with the appropriate treatment in order to reduce the physical and psychological stress associated with a diagnosis of cancer and treatment for cancer rather than a fungal infection. Also, if an initial diagnosis is a fungal infection rather than a malignancy, there is the potential to reduce the financial burden to healthcare. When patients present with clinical and radiologic findings that suggest the presence of a neoplasm, it is important to engage in astute differential diagnosis to determine a definitive diagnosis so that the appropriate treatment can be applied as soon as possible [6-8].

\section{Acknowledgement}

None.

\section{Conflict of Interest}

No conflict of interest.

\section{References}

1. Centers for Disease Control and Prevention (2017) Fungal diseases. Retrieved from https://www.cdc.gov/fungal/diseases/index.html

2. Shoham S, Levitz SM (2005) The immune response to fungal infections. Br J Haematol 129(5): 569-582.

3. Sayan M, Arpag H, Tokur M, Bahar AY (2018) Pulmonary mucormycosis mimicking an endobronchial mass. Asian Cardiovasc Thorac Ann 26(3): $242-244$.

4. World Health Organization (2018) Cancer. Retrieved from https://www. who.int/en/news-room/fact-sheets/detail/cancer

5. National Cancer Institute (2015) What is cancer?. Retrieved from https://www.cancer.gov/about-cancer/understanding/what-is-cancer

6. Gazzoni, FF, Severo LC, Marchiori E, Irion KL, Guimaraes MD, et al. (2014) Fungal diseases mimicking primary lung cancer: Radiologic-pathologic correlation. Mycoses 57(4): 197-208.

7. Guimaraes MD, Marchiori E, Godoy MCB (2013) Fungal infection mimicking lung cancer: A potential cause of misdiagnosis. American Journal of Roentgenology 201(2): W364-W364. Retrieved from https:// www.ajronline.org/doi/full/10.2214/AJR.13.10568

8. Guimaraes MD, Marchiori E, Meirelles G, Hochhegger B, Santana PRP, et al. (2013) Fungal Infection Mimicking Pulmonary Malignancy: Clinical and Radiological Characteristics. Lung 191(6): 655-652. 